\title{
Performance Analysis of Viterbi Decoder using different Digital Modulation Techniques in AWGN Channel
}

\author{
S. V. Viraktamath ${ }^{1}$, D. G. Talasadar ${ }^{2}$, Girish V. Attimarad ${ }^{3}$, G.A. Radder ${ }^{4}$ \\ ${ }^{124}$ (SDM College of Engineering and Technology, Dharwad, Karnataka, India.) \\ ${ }^{3}$ (Department of E\&CE, Dayanand Sagar College of Eng. Bangalore, Karnataka, India.)
}

\begin{abstract}
Error detection and correction has got a great practical importance in maintaining information (data) integrity across noisy channels. Convolutional encoding is considered as one of the forward error correction (FEC) schemes. A Viterbi decoder (VD) uses the Viterbi algorithm for decoding a bit stream that has been encoded using FEC based on a convolutional code. In this proposed work, a simulation of convolutional encoder and Viterbi decoder presented. This paper focuses on the performance study of Viterbi algorithm by varying parameters like constraint length, generating polynomials, traces back lengths and also modulation technique. The code rate considered is 1/2. In each case the Signal to Noise ratio $(S N R)$ is varied from -2 to 6 and the computation of Bit Error Rate (BER) is performed.
\end{abstract}

Keywords: FEC, Viterbi Decoder, Constraint length, Generator polynomial, Trellis diagram.

\section{INTRODUCTION}

The ever increasing use of wireless digital communication has led to a lot of effort invested in FEC. Wireless digital communication devices such as hand phones and broadband modems rely heavily on forward error correction techniques for their proper functioning, thus sending and receiving information with minimal or no error, while utilizing the available bandwidth. Viterbi algorithm is widely used in channel decoders for satellite and other noisy communication channels.

The main purpose of FEC is to improve the capacity of a channel by adding some carefully designed redundant information (redundant bits) to the data being transmitted through the channel [1]. Convolutional coding and block coding are the two major forms of channel coding. Convolutional codes operate on serial data, one or a few bits at a time [2]. Block codes operate on relatively large (typically, up to a couple of hundred bytes) message blocks. There are a variety of useful convolutional and block codes, and a variety of algorithms for decoding the received coded information sequences to recover the original data [3]. Convolutional encoding with Viterbi decoding is a FEC technique that is particularly suited to an AWGN channel. The Viterbi decoding algorithm was discovered and analyzed by Viterbi in 1967 [4]. It is used in decoding convolutional channel codes [5]. It is a robust technique that offers very high error resiliency at the expense of bandwidth. It basically implements maximum likelihood decoding rule, which states that the path traced in the trellis with minimum hamming distance must be the most likely path to contain the original data sequence. A number of decoding algorithms exist for decoding convolutional codes but Viterbi Algorithm is the most popular one [6]. Evaluation process of convolutional encoder and decoder (CODEC), for the various constraint lengths and for different generator polynomials is discussed considering only few bytes of data [7]. The performance analyzed for the burst errors and distributed errors. Impact of constrain length for the different input images for SOVA algorithm is presented in [8]. A high performance generic soft input hard output Viterbi decoder is presented [9] and prototyped on an FPGA board. The presented Viterbi decoder is intended to be used in a complete wireless LAN transceiver prototype.

Viterbi decoding method uses the Maximum Likelihood Decoding (MLD) algorithm, this method find a most likely pattern from the received data, and is known as the most optimum decoding method. In the proposed work the code rate $1 / 2$ is used. Modulation technique used for the transmission plays a very important role in communication. The quality of the signal received largely depends on the characteristics of the modulation technique like immunity to noise etc. This paper includes BER performance comparison of different modulation techniques like Phase Shift Keying (PSK), Quadrature Phase Shift Keying (QPSK). The performance of Convolutional CODEC is also analyzed for the different constraint lengths (CL) as well as for different generator polynomials.

\section{PROPOSED SYSTEM}

The model considered for the simulation is shown in Fig. 1. The channel considered is AWGN channel. At the receiver side two types of demodulations PSK and QPSK and Viterbi hard decision decoder are simulated. The different generator polynomials, different constraint lengths are considered for convolutional coding and decoding. The performance also analyzed by changing the trace back length. 


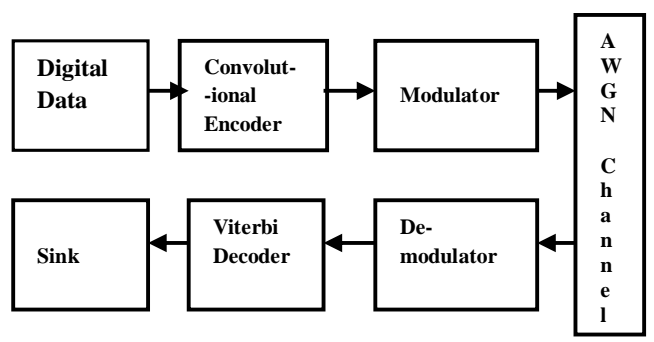

Fig. 1 Proposed system diagram

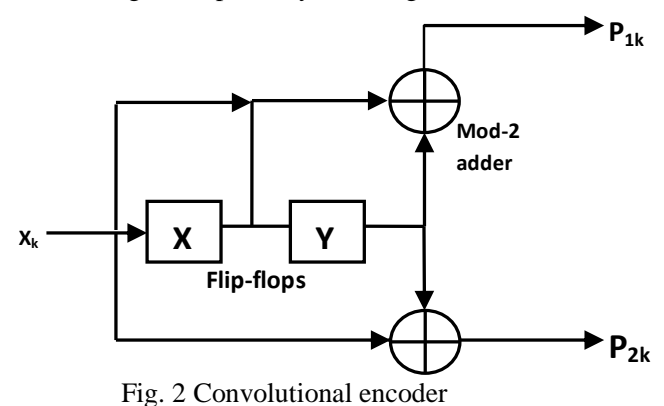

The performance measure parameter used is BER for different SNRs. Performance analysis is done in terms of BER for different constraint lengths, generator polynomials using different modulation techniques.

\section{The Simulation MODEL}

Convolutional codes are commonly specified by three parameters; $(n, k, m)$. Where ' $n$ ' is the number of output bits, ' $\mathrm{k}$ ' is the number of input bits considered at a time and ' $\mathrm{m}$ ' is the number of memory registers. The quantity $\mathrm{k} / \mathrm{n}$ called the code rate is a measure of the efficiency of the code. Convolutional encoder consists of a shift register with ' $m$ ' memory registers, and ' $n$ ' modulo-2 adders interconnected.

Convolutional encoding is accomplished using a combination of simple shift register and modulo-2 adders. In systematic style of encoding the input bits are included in the output stream along with the parity bits generated. Fig. 2 shows a $1 / 2$ rate non-systematic convolutional encoder with constraint length 3 . It makes use of two generator polynomials (g1, g2) for calculating two output bits (P1k, P2k) for every input bit (Xk) considered. Inputs to the mod-2 adder (which generates P1k, P2k) are decided by g1 and g2.

Using the next-state table and output table a trellis may be drawn as shown in Fig. 3. The Viterbi decoder makes use of this trellis diagram to calculate most probable input sequence. The zero input is represented by dotted line and one input is represented by the solid line in the Fig 3 . There are two branches leaving and entering each state. Each branch is labelled with the corresponding outputs. In general case of (n, $\mathrm{k}$, $\mathrm{m}$ ) code and an information sequence of length $\mathrm{kL}$, there are $2^{\mathrm{k}}$ branches leaving and entering each state, and $2^{\mathrm{kL}}$ distinct paths through the trellis corresponding to the $2^{\mathrm{kL}}$ code words. The decoder must produce the estimate of code word $\hat{V}$ of the code word $\mathrm{v}$ based on the received sequence $\mathrm{r}$. A MLD for a discrete memory less channel (DMC) chooses $\widehat{V}$ as the code word v which maximizes the log-likelihood function $\log \mathrm{P}(\mathrm{r} \mid \mathrm{v})$. Since for a DMC

$$
\log P(r \mid v)=\sum_{i=0}^{L+m-1} \log \mathrm{P}(\mathrm{ri} \mid \mathrm{vi})
$$

Where $\mathrm{P}$ (ri|vi) is a channel transition probability. The log-likelihood function $\log \mathrm{P}(\mathrm{r} \mid \mathrm{v})$ is called the metric associated with the path $\mathrm{v}$, and is denoted $\mathrm{M}(\mathrm{r} \mid \mathrm{v})$. The terms $\log \mathrm{P}(\mathrm{ri} \mid \mathrm{vi})$ in the sum (1) is called branch metrics and denoted by M(ri|vi). The Viterbi decoder algorithm works as follows:

1. Compute the partial metric for the single path entering each state in the Fig. 3 up to stage b.

2. Compute the partial metric for all the paths entering a state by adding the branch metric entering that state to the metric of the connecting survivors at the preceding time unit. For each state store the path with the largest metric (the survivor), together with its metric, and eliminate all other paths.

3. Repeat the step 2 for all $L+m$ stages. Stop

There are $2^{\mathrm{k}}$ survivors from time unit $\mathrm{m}$ through time unit ' $\mathrm{L}$ ', one for each of the $2^{\mathrm{k}}$ states. After time unit ' $L$ ' there are fewer survivors, since there are fewer states while the encoder is returning to the all-zero state. Finally, at time unit L+m, there is only one state, the all-zero state and hence only one survivor, and the algorithm terminates. After trellis construction is performed for the complete block of encoded data, trace back is performed for determining the decoded output sequence. The basic idea is to start from a specific state (pre- 
defined or having the smallest path metric) and trace the path leading to this state backwards in time to reconstruct the encoder input is a trace back length.

\section{RESULTS AND DISCUSSION}

The performance of Viterbi decoder is analysed by varying the constraint lengths, Generator polynomials, by changing the modulation techniques and by also varying the trace-back length.

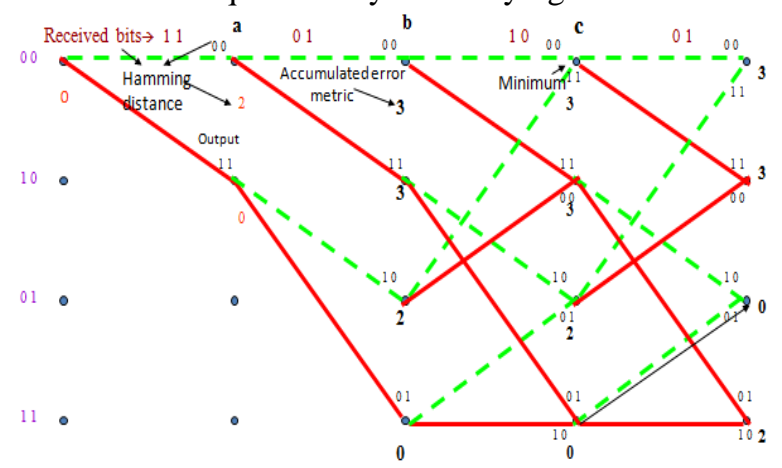

Fig. 3 Trellis diagram for Viterbi decoding algorithm

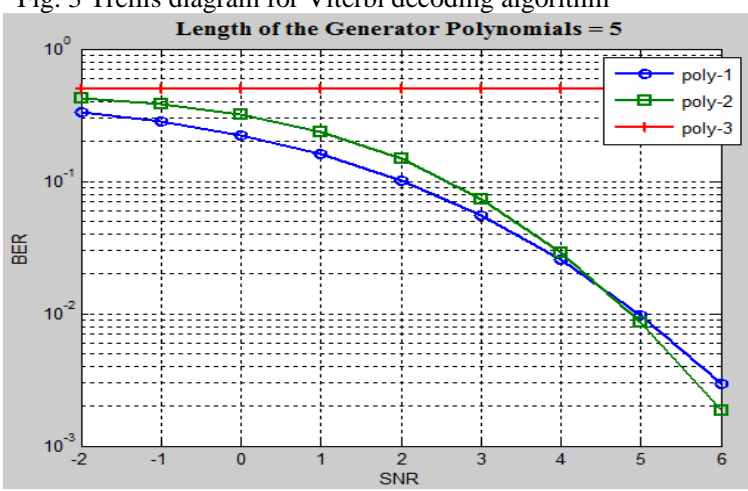

Fig. 4a Performance Analysis of VD for CL of 5

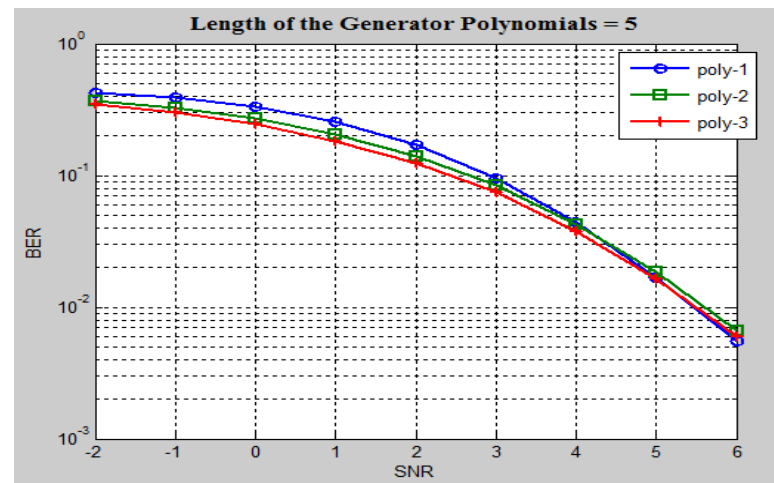

Fig. 4b Performance Analysis of VD for CL of 5

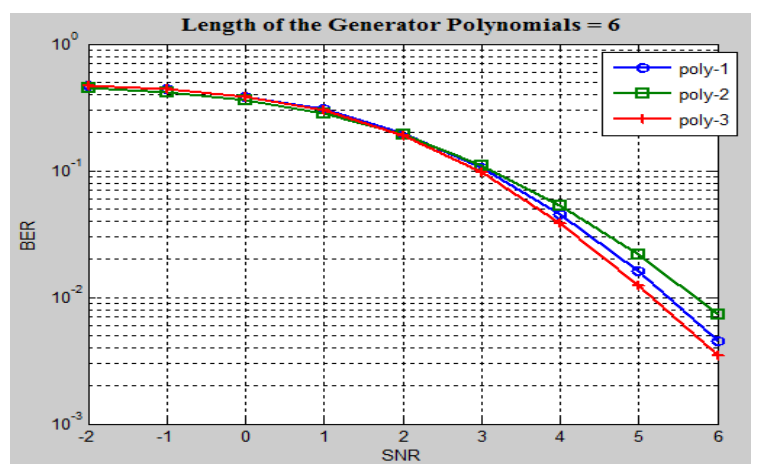

Fig. 5 Performance Analysis of VD for CL of 6 


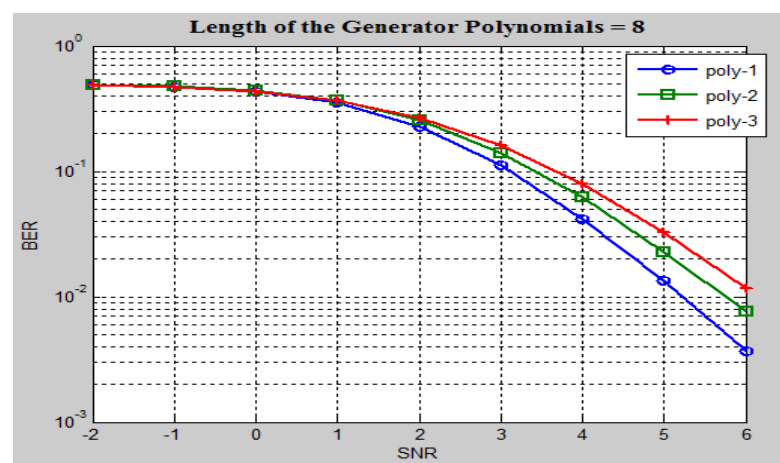

Fig. 6 Performance Analysis of VD for CL of 8

Fig. 4a and Fig. 4b shows the BER performance of VD for the constraint length of five. Fig. 4a also shows the performance for catastrophic polynomial. A decoder is said to exhibit catastrophic error-propagation when a finite number of channel errors results in infinitely many decoding mistakes. Three different generator polynomials with the constraint length of 5 are considered. The same set of inputs is considered for the three different polynomials simulation. It may be concluded that as the polynomial changes the BER performance also changes. BER performance for the constraint length of six and eight are shown in Fig. 5 and Fig. 6 respectively. While choosing the generator polynomial care should be taken to ensure that polynomial is not exhibiting catastrophic property.

From the simulation shown from Fig. 4 to Fig. 6 it may be concluded that the BER performance changes as the length of generator polynomial changes it also changes from polynomial to polynomial.

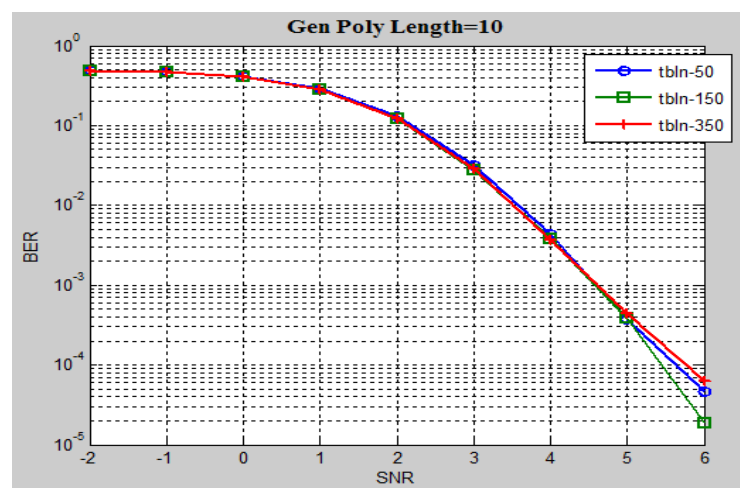

Fig. 7 Performance Analysis of VD for CL of 10 for different trace back lengths

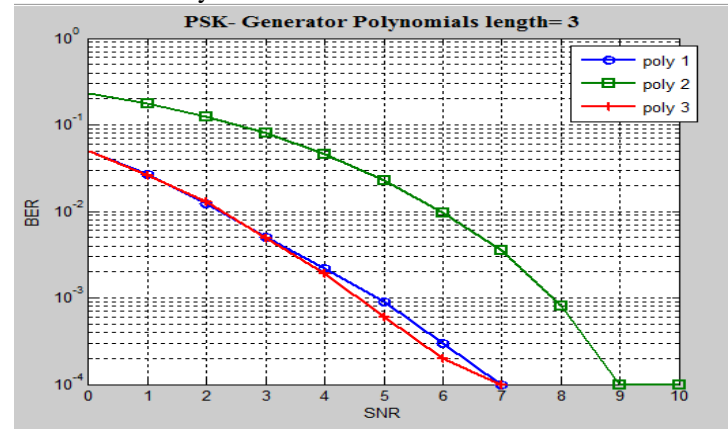

Fig.8 Performance Analysis of VD for PSK CL=3

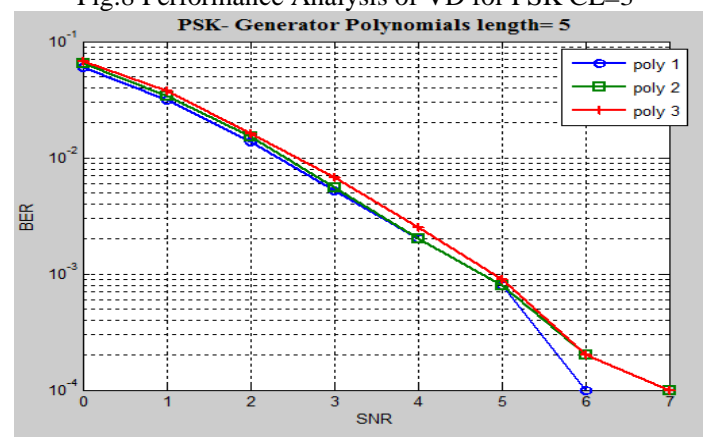

Fig.9 Performance Analysis of VD for PSK CL=5 
It is also observed that the decoding time increases as the length of the generator polynomial increases. The impact of trace back length also observed during the simulation. Fig. 7 shows the BER performance of Viterbi decoder for the constraint length of ten and for the trace back lengths of 50,150 and 350. The simulation shows that the BER performance is remaining same for lower SNRs, as the SNR is increasing small variations can be found. Fig. 8 and Fig. 9 shows the BER performance for the constraint length of three and five respectively for three different polynomials using the PSK modulation technique. The simulation is carried out for different constraint lengths. Fig. 10 shows the BER performance for the constraint lengths of 3, 4 and 5 for PSK. It may be concluded that performance improves as the constraint length increases. Fig. 11 shows the BER performance for the constraint lengths of 3,4 and 5 for the QPSK modulation technique. It may be concluded that performance improves as the constraint length increases.

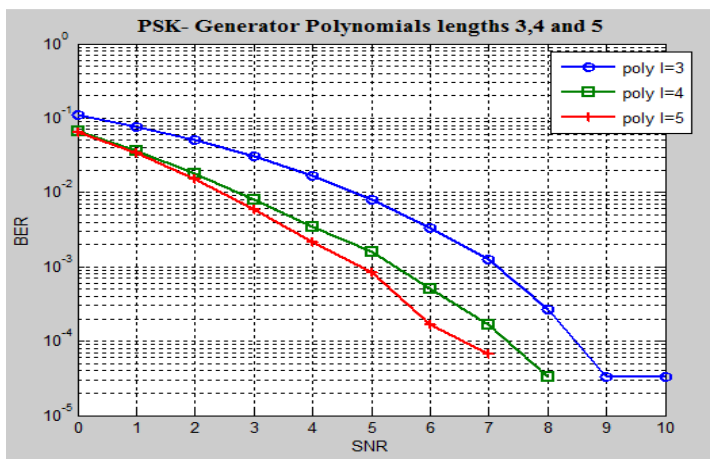

Fig.10 Performance comparison of VD for PSK

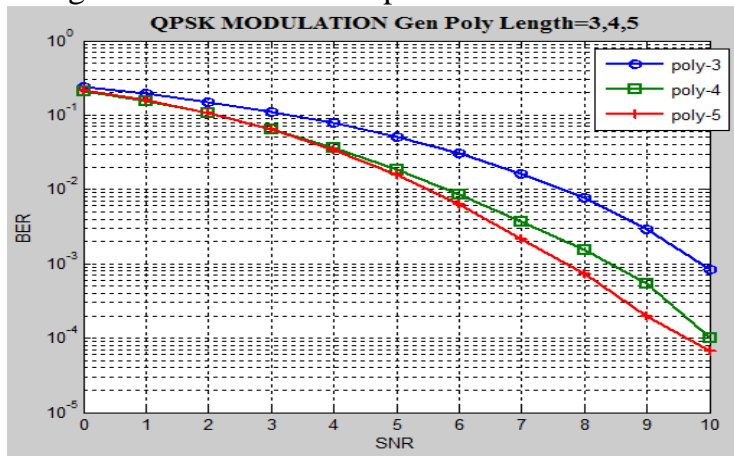

Fig.11 Performance comparison of VD for QPSK

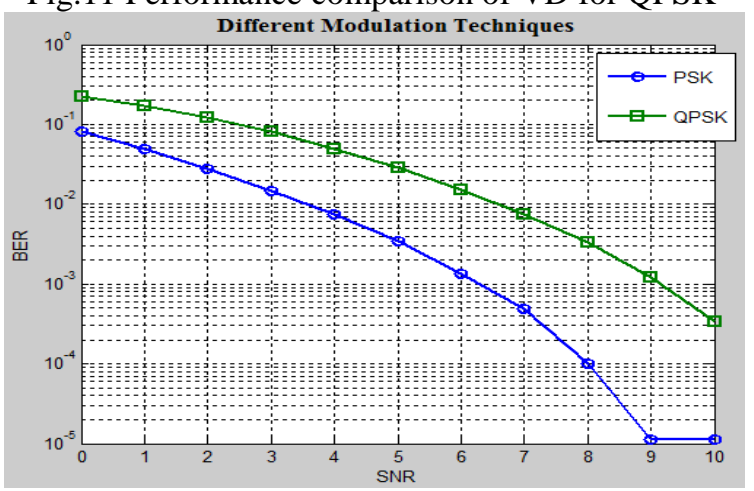

Fig.12 Performance comparison between PSK and QPSK

Fig. 12 shwos the BER performance comparison between the PSK and QPSK modulation techniques. The mathematical analysis shows that QPSK can be used either to double the data rate compared with a BPSK system while maintaining the same bandwidth of the signal, or to maintain the data-rate of BPSK but halving the bandwidth needed. From the Fig. 12 it may be concluded that the BER performance of PSK is better compared to QPSK.

\section{CONCLUSION}

In this paper, the effects of selection of constraint lengths, generator polynomials, trace back length and modulation techniques on the performance of a Viterbi decoder for convolutional codes has been investigated. By the simulation results it may be concluded that BER performance of Viterbi decoder changes as the constraint length and generator polynomials changes. The impact of selection of trace back length at lower SNR 
is very less. It is observed that the performance varies with change in generator polynomial even for the same constrained lengths. So a good performing generator polynomial for the given circumstances should be selected before proceeding. Depending upon the results obtained it may be concluded that the larger lengths of generator polynomials over perform the smaller lengths in terms of BER but increase the redundancy and in turn the bandwidth and memory requirements. From the results it may be concluded that the PSK modulation techniques give better performance compared to the QPSK modulation techniques in terms of BER.

\section{Acknowledgements}

An The authors thank the authorities of Sri Dharmasthala Manjunatheshwara College of Engineering and Technology, Dhavalagiri, Dharwad, and authorities of TEQIP 1.2 for encouraging us for this research work and providing financial support.

\section{REFERENCES}

[1] Bernard Sklar, Digital Communication Fundamentals and Application, 2001 pp. 314-374.

[2] Proakis, J. G., Digital Communication, McGraw-Hill Book Company, New York, Jan. 2000, pp 413-534.

[3] S. K. Hasnain, Azam Beg and M S Ghazanfar Monir, "Performance Analysis of Viterbi Decoder Using a DSP Technique", in 8th IEEE International Multi topic Conference (ITMIC'04), Lahore, Pakistan, 2004, pp. 201-207.

[4] V Viterbi A.J., "Error Bounds For Convolutional Codes And An Asymptotically Optimum Decoding Algorithm," IEEE Trans. Inf. Theory, vol. IT13, April 1967, pp.260-269.

[5] H Omura, J.K., "On the Viterbi Decoding Algorithm" (correspondence), IEEE Trans. Inf. Theory, vol. IT15 Jan. 1969, pp177-179.

[6] G. D. Forney Jr., "Convolutional codes I: Algebraic structure” IEEE Trans. Inform. Theory vol. IT-16 no. 6 pp. 268 - $278,1970$.

[7] S.V.Viraktamath, Dr.G.V.Attimarad, V.P.Gejji, Ravi. H "Error Control Mechanism Using CODEC", "International Conference on Communication Software and Networks 2009” (ICCSN 2009), February 27 - 28, 2009. Macau, China. Page No 549; 978-0-76953522-7/09 \$25.00 @ 2009 IEEE; DOI 10.1109/ICCSN.2009.142

[8] S.V.Viraktamath, Dr.G.V.Attimarad, "Impact of constraint length on performance of convolutional CODEC in AWGN channel for Image applications" has been published in "International Journal of Engineering Science and Technology (IJEST)", Vol. 2(9), 2010, 4697-4701. ISSN: 0975-5462

[9] Abdulfattah Mohammad Obeid, Alberto Garc'1a Ortiz, Ralf Ludewig and Manfred Glesner, "Prototyping of a High Performance Generic Viterbi Decoder", 13th IEEE International Workshop on Rapid System Prototyping (RSP'02).

Prof. S. V. Viraktamath. is with SDMCET, Dharwad, Karnataka, India. He is serving as Assistant Professor (S.G) in the Department of E\&CE. He has received a gold medal from VTU Belgaum for securing first rank in M.Tech (DC\&N). His research interests include Error control coding, Wireless communication and Networking.

Dr. G. V. Attimarad is with Dayanand Sagar College of Engineering Bangalore, Karnataka, India. He is serving as Professor in the Department of Electronics and Communication Engineering. His research interest includes area of waveguides and wireless communications. He has served as a reviewer for many International conferences.

Mrs. D. G. Talasadar is doing her M.Tech (CS) from SDM college of Engineering and Technology, Dharwad.

Prof. G.A. Radder is with the Dept. of Computer science in SDM college of Engineering and Technology, Dharwad. His area of interest is algorithms. 